\title{
PAPERS
}

\section{Impaired transit and tolerance of intestinal gas in the irritable bowel syndrome}

\author{
J Serra, F Azpiroz, J-R Malagelada
}

\begin{abstract}
Background-Patients with irritable bowel syndrome (IBS) frequently complain of excessive gas but their fasting volume of intestinal gas is apparently normal. We hypothesised that the pathophysiological mechanism involved may be impairment of intestinal gas transit.

Aim-To investigate intestinal gas transit and tolerance in IBS patients compared with healthy subjects.

Methods-A gas mixture $\left(\mathrm{N}_{2}, \mathrm{O}_{2}\right.$, and $\mathrm{CO}_{2}$ in venous proportions) was infused into the jejunum of 20 patients with IBS and 20 healthy controls at $12 \mathrm{ml} / \mathrm{min}$ for four hours. Gas evacuation, initially flatus from the anus (two hours) and then intrarectally (two hours), was continuously recorded. Symptom perception (0-6 scale) and abdominal distension were measured at 10 minute intervals.

Results-After two hours of external gas (flatus) collection, 18 of 20 IBS patients had developed gas retention $(>400 \mathrm{ml})$, increased gastrointestinal symptoms (score $>3$ ), or abdominal distension ( $>3$ mm girth increment) compared with only four of 20 control subjects. During intrarectal gas collection, 13 of 17 patients still exhibited abnormal responses.

Conclusion-A large proportion of patients with IBS can be shown to have impaired transit and tolerance of intestinal gas loads. This anomaly may represent a possible mechanism of IBS symptoms, specifically pain and bloating. (Gut 2001;48:14-19)
\end{abstract}

Digestive Syste
Research Unit,

Hospital General Vall

d'Hebron,

Autonomous

University of

Barcelona, Barcelona,

Spain

J Serra

F Azpiroz

J-R Malagelada

Correspondence to: Dr F Azpiroz, Digestive System Research Unit, Hospital General Vall

d'Hebron, 08035-Barcelona,

Spain.

azpirozf@hg.vhebron.es

Accepted for publication 8 August 2000 in the 1970 s by Levitt et al. These authors measured the volume of intestinal gas using an original washout technique ${ }^{12}$ and determined that patients with functional abdominal pain, which today would probably constitute IBS, have normal gas volumes in the gut. ${ }^{3}$ However, during the washout procedure performed by high rate infusion of argon, their patients presented abdominal symptoms and, in some, gas refluxed back into the stomach. The authors postulated that the primary problem in patients complaining of bloating and gas is abnormal gut motility. However, specific manometric and electromyographic studies in the subsequent two decades have failed to demonstrate abnormal motility patterns as the cause of IBS symptoms. ${ }^{4}$

In the present study we wished to extend these early original observations. Specifically, we hypothesised that patients with IBS have slow transit and evacuation of intestinal gas, which result in gas pooling and symptoms. In a group of IBS patients we measured not only endogenous gas volume but also intraabdominal transit of a gas load and its effects on abdominal perception and objective distension using a new technique developed and validated in our laboratory. ${ }^{5}$

\section{Material and methods}

PARTICIPANTS

Twenty patients with IBS (15 women, five men; age range 20-61 years) and 23 healthy individuals without gastrointestinal symptoms (14 women, nine men; aged 19-28 years) participated in the study after giving written informed consent. Healthy subjects were recruited by public advertising. Patients were recruited from the gastroenterology outpatient clinic and were selected on the presence of IBS diagnostic criteria, ${ }^{6}$ with no detectable abnormalities in routine testing and no concomitant diseases. Two patients refused to participate in the study. All patients were symptomatic at the time of study. Twelve patients were subjectively constipation predominant, four were diarrhoea predominant, and four alternated between constipation and diarrhoea. All but three patients, each from a different subset, complained of abdominal bloating. Bowel function was not assessed objectively nor was intestinal transit time of solids. The protocol for the study was previously approved by the Institutional Review Board of the Hospital General Vall d'Hebron.

Abbreviations used in this paper: IBS, irritable bowel syndrome; $\mathrm{SF}_{6}$, sulphurhexafluoride. 
JEJUNAL GAS INFUSION

We used an intestinal polyvinyl tube assembly (3.2 $\mathrm{mm}$ OD) that incorporated a gas infusion channel (1.6 mm ID) with multiple side holes over the $2 \mathrm{~cm}$ distal segment. A separate gastric polyvinyl tube (3.2 $\mathrm{mm}$ OD) was used to measure possible gaseous reflux/swallowing into the stomach.

A gas mixture containing $88 \%$ nitrogen, $6.5 \%$ carbon dioxide, and $5.5 \%$ oxygen bubbled into water for saturation was infused. These proportions were chosen to mimic the partial pressures of gases in venous blood and hence minimise diffusion across the intestinalblood barrier. ${ }^{7} 8$ A non-absorbable stable gaseous marker, $0.5 \%$ sulphurhexafluoride $\left(\mathrm{SF}_{6}\right)$, was added to the final mixture. ${ }^{9}$ Gas was infused continuously into the proximal jejunum at $12 \mathrm{ml} / \mathrm{min}$ using a modified volumetric pump (Asid Bonz PP 50-300; Lubratronics, Unterschleissheim, Germany).

MEASUREMENT OF GAS EVACUATION

Intestinal gas evacuation was measured either via an extra-anal cannula placed outside the anus to collect naturally passed flatus ${ }^{5}$ or via an intrarectal Foley catheter (20 French; Bard, Barcelona, Spain) with the balloon inflated with $5 \mathrm{ml}$ of water (see below). Anal or rectal gas was collected via a leakproof, low resistance collection line using a barostat, ${ }^{10}{ }^{11}$ and the volume automatically recorded on a paper polygraph (model 6006; Letica, Barcelona, Spain), as previously described. ${ }^{5}$ Subjects were provided with an event marker to signal every gas expulsion for later correlation with the volume collection recording.

MEASUREMENTS OF ABDOMINAL GIRTH CHANGES Once subjects were in bed (see below), a nonstretch $48 \mathrm{~mm}$ wide belt with a metric tape measure was adjusted around the abdomen over the umbilicus by means of two elastic bands. Girth measurements were taken while the subjects were breathing in a relaxed manner as the average of inspiratory and expiratory determinations over three consecutive respiratory excursions.

\section{PERCEPTION QUESTIONNAIRE}

A graded questionnaire was used to measure the intensity and type of sensations perceived, and an anatomical questionnaire to measure the location and extension of the perceived sensations. ${ }^{512-14}$ The graded questionnaire, which included four graphic rating scales graded from 0 (no perception) to 6 (pain), was used to specifically score: (a) abdominal pressure/bloating, (b) colicky sensation, (c) stinging sensation, and (d) other types of sensation (to be specified), respectively. The questionnaire included two additional scales for scoring the feeling of difficult gas evacuation from the anus and belching, respectively, which were analysed separately. Participants were asked to score any perceived sensation (one or more perceived simultaneously) on the scales and to tick a box (yes/no) to indicate whether the sensations were identified as their usual clinical complaints.
The anatomical questionnaire incorporated a diagram of the abdomen divided into nine regions corresponding to the epigastrium, periumbilical area, hypogastrium, both hypochondria, flanks, and iliac fossae. Participants were instructed to mark the location-that is, abdominal region(s) or extra-abdominalwhere the sensations were perceived.

\section{PROCEDURE}

Participants were instructed to follow a diet excluding legumes, vegetables, garlic, onions, nuts, cereals, wholemeal bread, and fizzy drinks during the two days prior to the study. The night before the study they had a light dinner that consisted of meat, fish, eggs, rice, pasta and/or white bread, but avoiding dairy products, salad, fruit, and alcoholic beverages. All medication was discontinued one week before the study. On the day of the study participants were orally intubated after an eight hour fast. The tip of the intestinal tube assembly was positioned $5 \mathrm{~cm}$ caudal to the angle of Treitz under fluoroscopic control. The studies were conducted in a quiet room with the subjects placed supine on the bed at a $30^{\circ}$ angle. A 30 minute equilibration period was allowed before starting intestinal gas infusion and collection. During the study period intestinal gas was continuously infused and gas evacuation recorded; a sample of gas evacuated (flatus) for every 30 minute period was stored in metallised bags (Gas collection $750 \mathrm{ml}$; QuinTron, Milwaukee, Wisconsin, USA) for later analysis. Perception and abdominal girth were measured at 10 minute intervals.

\section{EXPERIMENTAL DESIGN}

Main studies

In 20 healthy subjects and 20 IBS patients, intestinal gas was continuously infused into the intestine and flatus collected via the extra-anal cannula for two hours. Unless otherwise stated, data in the results section refer to the anal collection period.

\section{Effect of rectal intubation}

The study was continued in 10 healthy subjects and 17 IBS patients to test the potential role of anal retention. Without interruption of the gas infusion, the collection cannula was changed and gas collected via the intrarectal catheter for an additional two hour period.

\section{OUTCOME MEASURES}

In each subject, the volume of gas retained at different time periods within the gut was calculated as the difference between the volume of gas infused and the volume of gas recovered. Over the same time periods, $\mathrm{SF}_{6}$ concentration in the gas evacuated was measured by infrared absorbance after determination of standard curves. ${ }^{15}$ Based on $\mathrm{SF}_{6}$ recovery (volume of gas collected $\times \mathrm{SF}_{6}$ concentration) the volume of exogenous gas recovered was calculated. This value was subtracted from the total volume of gas evacuated to calculate the volume of endogenous gas evacuated.

Perception during the studies was measured by the score in the graded questionnaire. When 


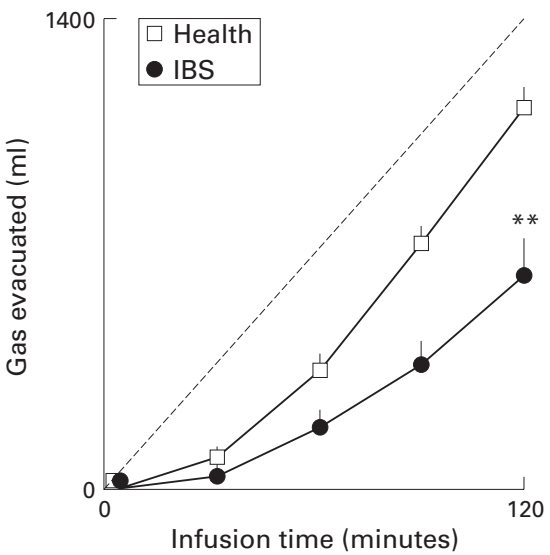

Figure 1 Evacuation of intestinal gas in patients with irritable bowel syndrome (IBS) $(n=20)$ and healthy subjects $(n=20)$. Gas was infused into the intestine at a constant rate (represented by the broken line) for two hours and collected via an anal cannula. Note that IBS patients expelled a significantly lower volume of gas. Values are means (SEM). ${ }^{\star *} p<0.01$.

more than one sensation was simultaneously scored, only the maximum score, instead of the cumulative score, was computed for comparisons. In each subject, the number of times each sensation was scored was counted to calculate the frequency (as per cent distribution) of each specific sensation. In the anatomical questionnaire, the percentage of stimuli referred to over more than one abdominal region was calculated.

Changes in abdominal girth throughout the study were referred to measurements taken at the beginning of the study before gas infusion.

\section{STATISTICAL ANALYSIS}

Mean (SEM) values of the parameters were calculated in each group of subjects. Mean gas retention, perception scores, and changes in abdominal girth in IBS patients were compared with those of healthy controls using the Mann-Whitney U test. Within each group, parameters during anal and rectal gas collection were compared using the paired Wilcoxon signed rank test. Contingency tables of both groups were analysed by Fisher's exact test. Correlations of paired data were examined by linear regression analysis.

VALIDATION STUDIES

Reproducibility

A second study, separated by an interval of 2-8 months, was conducted in five healthy subjects following the same procedure as in the main studies - that is, two hour intestinal gas infusion and anal collection. In the first and second studies, data on intestinal gas retention (157 (10) $\mathrm{ml}$ and 171 (28) $\mathrm{ml}$, respectively), perception score $(1.5(0.7)$ and $1.8(0.2)$, respectively) and girth increment $(0$ (2) $\mathrm{mm}$ and 2 (1) $\mathrm{mm}$, respectively) showed very good reproducibility.

\section{Gas recovery}

An additional study was performed in five healthy subjects infusing $5 \% \mathrm{SF}_{6}$ only during the first 10 minute period but otherwise following the same procedure as in the main experiments. After two hours of anal gas collection, 95 (2)\% $\mathrm{SF}_{6}$ was recovered.

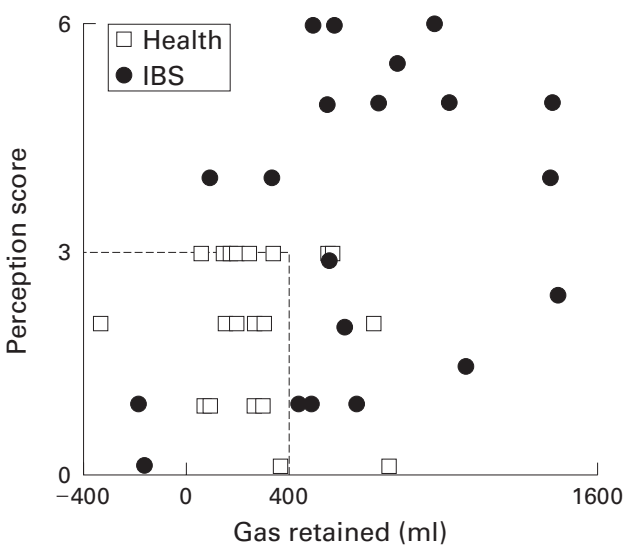

Figure 2 Individual perception scores and gas retention after a two hour intestinal gas infusion in patients with irritable bowel syndrome (IBS) and healthy subjects. Perception of abdominal symptoms was scored on a seven point scale (0-6). Gas retained is volume infused minus volume evacuated. Broken lines represent the upper limits for perception (score 3-that is, mild to moderate) and gas retention ( $400 \mathrm{ml}$, see text) in healthy subjects. Only 4/20 healthy subjects progressively retained gas exceeding 400 ml. In contrast, most IBS patients $(18 / 20)$ retained gas $(>400 \mathrm{ml}$ ) and/or developed moderate to severe symptoms ( $>3$ perception score).

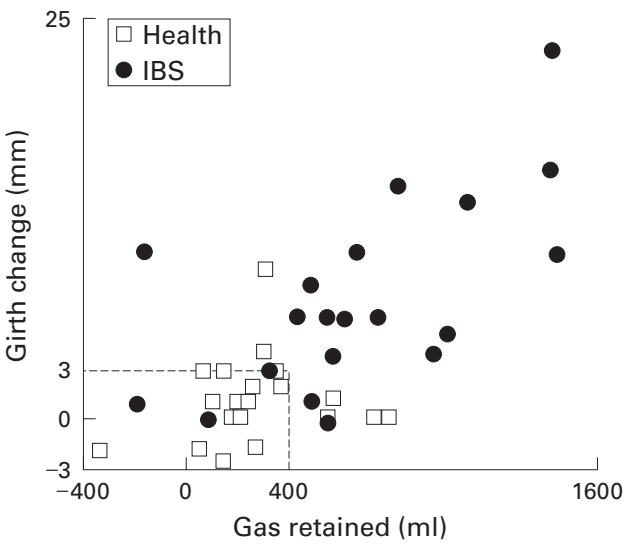

Figure 3 Individual abdominal distension and gas retention after a two hour intestinal gas infusion in patients with irritable bowel syndrome (IBS) and healthy subjects. Gas retained is volume infused minus volume evacuated. Note that in contrast with healthy subjects, most IBS patients (17/20) retained gas $(>400 \mathrm{ml})$ and/or developed abdominal distension ( $>3 \mathrm{~mm}$ girth increment).

\section{Results}

GAS EVACUATION

Most healthy subjects adequately handled the $12 \mathrm{ml} / \mathrm{min}$ gas load, and gas evacuation rates paralleled gas infusion rates with no substantial intra-abdominal gas retention (fig 1). As in previous studies, ${ }^{5}$ only a small proportion of healthy subjects presented progressive gas retention during gas infusion and only in those did retention exceed $400 \mathrm{ml}$ after a two hour infusion period. Hence an upper limit of gas retention of $400 \mathrm{ml}$ was chosen to define gas retainers. In IBS patients, gas retention at the end of the two hour period was significantly greater than in healthy controls $(667(104) \mathrm{ml} v$ 273 (56) $\mathrm{ml} ; \mathrm{p}<0.01$ ) (fig 1 ). The proportion of gas retainers, defined as those with progressive gas retention exceeding $400 \mathrm{ml}$ after two hours, was significantly higher among IBS patients than healthy subjects $(\mathrm{p}<0.01)$ (figs 2,3$)$. 
The correlation between reported and recorded flatus evacuation was excellent in all participants (99 (1)\% of the reported evacuations were recorded; pooled data). Reduced evacuation in IBS patients was associated with a decreased number of flatus passages (12 (2) in IBS $v 21$ (2) in healthy subjects; $p<0.01$ ) but mean gas volume per flatus was similar in both groups (52 (7) $\mathrm{ml}$ in IBS and 61 (6) $\mathrm{ml}$ in healthy subjects). Mean gas retention was 727 (70) $\mathrm{ml}$ in constipation predominant IBS patients, $572(70) \mathrm{ml}$ in diarrhoea predominant, and 583 (32) $\mathrm{ml}$ in patients alternating between constipation and diarrhoea; these values were not significantly different. Gastric gas reflux was detected in only three IBS patients who failed to tolerate the procedure, just immediately before vomiting and interruption of the gas infusion (see below).

INTENSITY OF PERCEPTION

In healthy subjects, the intestinal gas load induced relatively low abdominal perception and no subject perceived a score above 3 (average score $2.0(0.2)$ ) (fig 2). IBS patients reported higher perception (average score 3.5 $(0.4) ; \mathrm{p}<0.05 v$ healthy controls). Furthermore, more than half of IBS patients reported perception scores above 3, seven experienced frank discomfort, and the study was discontinued in three patients after 50-90 minutes of infusion because of abdominal pain, nausea, and vomiting. The correlation between gas retention and abdominal perception was fair $(r=0.42 ; \mathrm{p}<0.01)$. Thus some IBS patients developed symptoms with retained gas volumes that were well tolerated by healthy subjects. Considering the combination of gas retention and perception, 18 of 20 IBS patients fell outside the normal range $(\mathrm{p}<0.01 v$ healthy subjects) (fig 2).

NATURE OF SYMPTOMS

During intestinal gas infusion, mild abdominal symptoms reported by healthy subjects consisted of colicky sensation, pressure/bloating, and stinging sensation $(64(6) \%, 22(6) \%$, and 13 (5)\%, respectively). Patients with IBS reported a similar symptom pattern, albeit more severe. Eleven of 20 IBS patients frequently (on 71 (11)\% of occasions) recognised the perceived sensations as their usual clinical symptoms. Eight IBS patients with gas retention exceeding $400 \mathrm{ml}$ described feelings of difficult gas evacuation. Only three IBS patients who were unable to complete the study presented belching just before interruption.

Both healthy subjects and IBS patients referred their symptoms to the abdominal midline (27 (5) \% of symptoms were referred to the epigastrium, $50(6) \%$ to the periumbilical area, and 48 (6)\% to the hypogastrium; pooled data for healthy and IBS patients). The extent of the referral area tended to be greater in IBS patients but the differences detected did not reach statistical significance (65 (6)\% of sensations were perceived over more than one area in IBS patients $v 51$ (7) $\%$ in controls).
ABDOMINAL DISTENSION

The belt used for girth measurements remained in place and permitted automated measurements over time. Girth measurements showed good reproducibility in consecutive determinations (coefficient of variation $<1 \%$ ). During infusion of gas, abdominal girth changed by 1 (1) $\mathrm{mm}$ in healthy subjects and by $7(1) \mathrm{mm}$ in IBS patients $(\mathrm{p}<0.01)$. Fifteen IBS patients in contrast with only two healthy subjects $(p<0.01)$ developed abdominal distension greater than $3 \mathrm{~mm}$ during intestinal gas infusion (fig 3). The correlation between gas retention and abdominal distension was relatively high $(r=0.66 ; \mathrm{p}<0.001)$.

ROLE OF THE ANUS

Gas retention decreased during the second part of the study with rectal collection, and this effect was similar in healthy subjects and IBS patients: at the end of the two hour period, gas retention had decreased by $364(82) \mathrm{ml}$ and 364 (113) $\mathrm{ml}$, respectively. Only two IBS patients evacuated large volumes of gas after rectal intubation ( $>600 \mathrm{ml}$ in 10 minutes) with improved symptom perception. However, during the period of the study with intrarectal cannula, IBS patients still presented significantly more gas retention than healthy subjects $(302$ (127) $\mathrm{ml} v-110$ (127) $\mathrm{ml}$, respectively; $\mathrm{p}<0.05)$. After two hours of rectal collection, IBS patients reported a mean perception score of $3.5(0.5)$ compared with $2.0(0.4)$ in healthy subjects; girth increment was 7 (3) $\mathrm{mm}$ in IBS patients compared with -1 (1) $\mathrm{mm}$ in healthy subjects $(\mathrm{p}<0.05$ for both parameters $)$. Pooling retention $(<400 \mathrm{ml})$, perception (<score 3$)$, and distension ( $<3 \mathrm{~mm}$ ), 13 of 17 IBS patients but only two of 10 healthy subjects fell outside the normal range during intrarectal gas collection $(\mathrm{p}<0.01)$.

\section{ENDOGENOUS VERSUS EXOGENOUS GAS}

EVACUATION

The amount of endogenous gas evacuated during the 240 minute gas infusion period was similar in healthy subjects and IBS patients. Exogenous gas, labelled with $\mathrm{SF}_{6}$, started to be evacuated after a time lag, the concentration in the external collection increased thereafter and then plateaued from 180 minutes onwards. The increase in exogenous gas concentration was significantly slower in IBS patients than in healthy subjects: for example, at one hour the concentration was $2(1) \%$ in IBS patients compared with 19 (4)\% in healthy subjects $(\mathrm{p}<0.05)$. Based on the $\mathrm{SF}_{6}$ concentration in the last two sample measurements (after steady state had been reached) the volume of endogenous gas produced during the study was calculated. This value was then subtracted from the total volume of endogenous gas evacuated to obtain the volume of endogenous gas present in the intestine at the beginning of the experiment. This volume did not differ significantly in IBS patients (287 (41) ml) compared with healthy subjects (199 (43) ml). 


\section{Discussion}

By applying our new technique to quantitate intestinal gas transit and tolerance, we have shown that impaired transit of infused gas loads results in intra-abdominal gas pooling and induces symptoms that many IBS patients recognise as their own.

Impaired intestinal gas transit in IBS may result from altered gut motor function but the precise site and mechanism of the abnormality cannot be deduced from the present study. Potentially, gas pooling may result from either defective gas propulsion (impaired phasic activity), increased resistance to gas flow (uncoordinated phasic activity), or increased capacitance of gas volumes (defective tone). However, the latter seems unlikely as intestinal gas accumulation, induced via pharmacological relaxation using intravenous glucagon, causes distension but no sensory symptoms. ${ }^{16}$ Nevertheless, the intestinal motor activity that moves gas remains unknown and appears to be independent of chyme-faecal propulsion as large quantities of gas were evacuated during the study without a call for stools.

Conscious suppression of flatus passage may produce intestinal gas pooling and symptoms. ${ }^{16}$ Hence in the present study we also investigated the specific role of anal sphincter activity in gas retention by comparing responses to the intestinal gas load in individuals fitted with an intrarectal or external gas collection device. Our data showed that gas pooling due to anal retention was a prominent pathophysiological mechanism in two IBS patients who drained large volumes of gas and became symptom free shortly after rectal intubation. An additional subset of IBS patients exhibited some improvement during rectal collection. Hence failure to expel gas via the anus (an activity partially under voluntary and "social" control) may play a pathophysiological role in IBS. Nevertheless, the majority of IBS patients still presented abnormal responses to the gas load during the two hour gas infusion and rectal gas collection period, specifically gas retention, abdominal symptoms, and/or distension. These data may suggest that a more proximal mechanism slows gas transit in a proportion of IBS patients and may thus provide indirect evidence of intestinal dysmotility in IBS not detectable by conventional techniques. ${ }^{4}$ However, we wish to acknowledge that gas retained by an anal mechanism could have refluxed back into the proximal colon and consequently our results should be interpreted with caution.

In our study, abdominal distension correlated with gas retention. Distension, a frequent clinical complaint in IBS, has previously been investigated without much success. ${ }^{17-19}$ Excess gas was a hypothesis specifically ruled out in earlier studies ${ }^{3}$ and our data concur with the main conclusion: there is no basal increase in intra-abdominal gas in IBS. However, intubation studies were performed in the fasting early morning period when IBS patients usually complain of neither distension nor other abdominal symptoms; in most patients these symptoms develop or increase during the day as meals, physical activity, and stress accumu- late. Indeed, gas evacuation, and conceivably gas production, is greater during the day, particularly in the postprandial period after consumption of flatulogenic foodstuffs. ${ }^{2021}$ Furthermore, IBS patients have recently been reported to have abnormal colonic fermentation and increased gas production. ${ }^{22}$ This abnormality "per se" would not justify IBS complaints as gas loads in the same range are well tolerated by healthy subjects. ${ }^{52}$ Therefore, both increased gas production and impaired gas transit must interact to produce symptoms.

Our contention is that as gas transit is altered gas would tend to pool inside the gut and clinical manifestations arise. Some of our patients complained of symptoms without abnormal volumes of gas retention, and others even showed normal responses to gas loads. Individual variations in gas distribution or gut compliance could explain such a dissociation between symptoms and gas retention in some individuals. Furthermore, perception of intraabdominal gas may be modified by visceral hypersensitive responses to wall tension that have been well documented in IBS patients, ${ }^{23} 24$ thereby augmenting perception of increased and even normal amounts of intestinal gas. In this respect, spatial summation phenomena ${ }^{1425}$ become particularly relevant as gas would tend to spread along the intestine and stimulate a large number of mechanosensitive afferents.

Our method of measuring gas transit and tolerance has been validated in detail both in the present and previous studies. ${ }^{5}$ We have shown that the gas collection system is hermetic, gas retention calculations prove reliable when challenged against the standard washout technique, ${ }^{1}$ and the results are reproducible. Using this technique we identified, in previous and in the present study, a small proportion of the normal population with reduced capacity to evacuate intestinal gas loads in the absence of complaints in their everyday life. ${ }^{5}$ Hence it is conceivable that patients with IBS have a more pronounced defect which results in clinical symptoms. We acknowledge the artificial nature of our experiments and the selection of patients but our present results necessitate reconsideration of the role of intestinal gas in IBS. It may not be "too much gas", but transit and evacuation of intestinal gas may be impaired in a large proportion of IBS patients and become a potential mechanism for symptoms such as pain and bloating, that can no longer be ignored.

Supported in part by the Spanish Ministry of Education (Dirección General de Enseñanza Superior del Ministerio de Educación y Cultura, PM97-0096). Dr Serra was supported by a scholarship from the Spanish Ministry of Education (CICYT). The authors thank Nuria Ferrer and Isidre Casals, Serveis Cientifico-Tecnics of the Central University of Barcelona for help in gas infrared absorbance analysis; Maite Casaus and Anna Aparici for technical support; Gloria Santaliestra and Montse Domenech for secretarial assistance; and Ms Christine O'Hara for English language editing of the manuscript.

1 Levitt MD. Volume and composition of human intestinal gas determined by means of an intestinal washout technic. $N$ Engl f Med 1971;284:1394-8.

2 Levitt MD, Bond JH. Volume, composition and source of intestinal gas. Gastroenterology 1970;59:921-9.

3 Lasser RB, Bond JH, Levitt MD. The role of intestinal gas in functional abdominal pain. $N$ Engl f Med 1975;293:524-6. 
4 Drossman DA, Whitehead WE, Camilleri M. Irritable bowel syndrome: a technical review for practice guideline

5 Serra J, Azpiroz F, Malagelada J-R. Intestinal gas dynamics and tolerance in humans. Gastroenterology 1998;115:54250.

6 Thompson WG and the Working Team for Functional Bowel Disorders. C. Functional bowel disorders and D. Functional abdominal pain. In: Drossman DA, Richter JE, Talley NJ, et al, eds. The functional gastrointestinal disorders: diagnosis, pathophysiology and treatment. Boston, MA: Little, Brown and Company, 1994:115-73.

7 Foster RE. Physiological basis of gas exchange in the gut. Ann N Y Acad Sci 1968;150:4-12.

8 Pogrund RS, Steggerda FR. Influence of gaseous transfer between the colon and blood stream on percentage gas compositions of intestinal flatus in man. Am ₹ Physiol 1948; 153:475-82.

9 Levitt MD. Production and excretion of hydrogen gas in man. N Engl f Med 1969;281:122-7.

10 Azpiroz F, Malagelada J-R. Physiologic variations in canine Azpiroz $\mathrm{F}$, Malagelada J-R. Physiologic variations in canine
gastric tone measured by an electronic barostat. $A m \mathcal{F}$ gastric tone measured by

11 Azpiroz F, Malagelada J-R. Gastric tone measured by an electronic barostat in health and postsurgical gastroparesis. Gastroenterology 1987;92:934-43.

12 Azpiroz F. Sensitivity of the stomach and the small bowel: human research and clinical relevance. In: Gebhart GF, ed. Progress in pain research and management, vol. 5. Visceral Pain. Seattle, Washington: IASP, 1995:391-428.

13 Gracely RH. Studies of pain in normal man. In: Wall PD, Melzac R, eds. Textbook of pain, 3rd edn. Edinburgh: Churchill Livingstone, 1994:315-36.

14 Serra J, Azpiroz F, Malagelada J-R. Perception and reflex responses to intestinal distension in humans are modified by simultaneous or previous stimulation. Gastroenterology 1995;109:1742-9.
15 Jonmarker C, Castor R, Drefeldt B, et al. An analyzer for in-line measurement of expiratory sulfur hexafluoride concentration. Anesthesiology 1985;63:84-8.

16 Serra J, Azpiroz F, Malagelada J-R. Gas symptoms depend on the cause of retention: impaired gut propulsion vs obstructed evacuation. Neurogastroenterol Mot 1998;10:96.

17 Chami TN, Schuster MM, Bohlman ME, et al. A simple radiologic method to estimate the quantity of bowel gas. Am f Gastroenterol 1991;86:599-602.

18 Maxton DG, Martin DF, Whorwell PJ, et al. Abdominal distension in female patients with irritable bowel syndrome: exploration of possible mechanisms. Gut 1991;32:662-4.

19 Poynard T, Hernandez M, Xu P, et al. Visible abdominal distension and gas surface: description of an automatic method of evaluation and application to patients with irritable bowel syndrome and dyspepsia. Eur 7 Gastroenterol Hepatol 1992;4:831-6.

20 Tomlin J, Lowis C, Read NW. Investigation of normal flatus production in healthy volunteers. Gut 1991;32:665-9.

21 Kirk E. The quantity and composition of human colonic flatus. Gastroenterology 1949;12:782-94.

22 King TS, Elia M, Hunter JO. Abnormal colonic fermentation in irritable bowel syndrome. Lancet 1998;352:1187-9.

23 Whitehead WE, Engel BT, Schuster MM. Irritable bowel syndrome: physiological and psychological differences syndrome: physiological and psychological differences
between diarrhea-predominant and constipationpredominant patients. Dig Dis Sci 1980;25:404-13.

24 Accarino AM, Azpiroz F, Malagelada J-R. Selective dysfunction of mechanosensitive intestinal afferents in the irritable bowel syndrome. Gastroenterology 1995;108:63643.

25 Serra J, Azpiroz F, Malagelada J-R. Modulation of gut perception in humans by spatial summation phenomena. f Physiol (Lond) 1998;506:579-87. 